\title{
Surface energy and its anisotropy of hexagonal close-packed metals
}

\author{
Yongkun Luo*, Rongshan Qin* \\ Department of Materials, Imperial College London, SW7 2AZ, London, United Kingdoms
}

\begin{abstract}
The absolute unrelaxed surface energy and its full orientation-dependent behaviour of $13 \mathrm{HCP}$ metals are studied via a broken-bond base geometric model. The model is integrated with the Rose-Vinet universal potential to investigate arbitrary orientations which are not assessable by other methods. Using only three materials constants, the calculated results show only marginal discrepancies with reported experimental values, except for divalent sp metals $\mathrm{Mg}$, Zn and Cd where the calculated values are lower by a factor of 2. Stereographic projections of all 13 metals show global minimum on (0001) pole with an overall anisotropy of $15 \%$ to $21 \%$. The equilibrium crystal shape of HCP is found to be truncated hexagonal bi-prismatic, with (0001) always favoured but the bi-prismatic facets vary from one metal to another. All projection patterns show strong six-fold symmetries but are unique for every element. The patterns are found to be largely determined by an anharmonicity factor $\eta$. Best agreement with experimental findings are found for Be, Sc, Ti,Y, Zr and Hf which possess comparatively low $\eta$. We believe the stereographic projections of these elements are the more representative for HCP metals.
\end{abstract}

Keywords: surface energy; surface energy anisotropy; HCP metals; equilibrium crystal shape

\footnotetext{
*Corresponding author

Email addresses: yongkun.luo08@imperial.ac.uk (Yongkun Luo), r.qin@imperial.ac.uk (Rongshan Qin)
} 


\section{Introduction}

Being one of the most important fundamental quantity in the context of surface science, surface energy enjoys a vast influence on the properties of both structural and functional materials as it controls how a surface rethe surface energy of crystalline substances exhibits orientation-dependent behaviour originated from their long-range structural order. The surface energy anisotropy of a substance is largely associated with its corresponding lattice structure, but also sensitively dependent on the physical and chemical standing of surface energy and its anisotropy enables manipulation of not only the properties of crystalline functional materials such as absorption and catalytic capacity, but also the macroscopic physical properties of polycrystalline structural materials such as toughness and fatigue resistance. the best experimental approach consists of analysis of carefully equilibrated crystallites $[1,2,3]$. Such process is viable on some materials but not the others, and can be questionable in reproducibility. As such, a large portion of modern understanding on the topic of surface energy is obtained via computational methods, such as density-function theories (DFT) or embeddedatom methods (EAM) type semi-empirical approaches $[4,5,6,7,8,9,10,11$, $12,13,14,15,16,17]$. However, the computational costs of these methods raise dramatically for atomically complex surfaces and thus render them less suitable for the investigation of the full anisotropy of surface energy.

Being regarded as brittle materials thus overlooked in the past, hexagonal close-packed (HCP) metals attract a recent resurgence of interests owing to some of the unique properties they possess. Nonetheless, compared to cubic metals, the knowledge regarding HCP metals in the context of surface energy, particularly its anisotropy, is somewhat limited. Following our latest success on face-centred cubic (FCC) metals [18], in the present article, an improved version of Mackenzie's original broken-bond model is introduced for the HCP metals. Combined with the Rose-Vinet universal potential equation, the new model is used to provide the absolute unrelaxed surface energy and its full orientation-dependence of eleven HCP metals. Only three input parameters, namely the lattice constant, the bulk modulus and the cohesive energy is used in the process. The method allows construction of three-dimensional $\gamma$ plots which lead to many new interesting understanding of the subject. The 
naturally favoured facets of HCP metals are revisited base on these findings. The remainder of this paper is organized as follows. Section 2 introduces the theoretical parts of our model. Results and discussions are presented in Section 3. Finally Section ?? concludes our work.

\section{Theory}

According to Herring [19], the excessive energy of surface with orientation $\hat{\mathbf{n}}$ can be expressed as

$$
\Gamma(\hat{\mathbf{n}})=\frac{1}{2} \sum f_{\mathbf{b}}(\mathbf{b}) \phi_{\mathbf{b}}
$$

where $\phi_{\mathbf{b}}$ denotes the energetic contribution from a two-body type interaction between atoms linked by vector $\mathbf{b}$, and $f_{\mathbf{b}}$ is the amount of $\mathbf{b}$ type bonds broken upon the creation of a pair of such surface. It is generally questionable whether such two-body formalism is physically representative for metallic systems. Alternatively, an EAM type many-body expression for surface energy is given as follows [20]:

$$
E_{(x)}^{f}=\sum \frac{E_{c} \frac{Z_{i}-Z_{d}}{Z_{i}}+F_{i} \frac{\bar{\rho}_{i}^{x}}{Z_{i}}-\frac{Z_{d}}{Z_{i}} \cdot F_{i} \frac{\bar{\rho}_{i}{ }^{0}}{Z_{i}}}{A_{(x)}}
$$

where $E_{c}$ is the cohesive energy of reference lattice; $\bar{\rho}_{i}{ }^{x}$ and $\bar{\rho}_{i}{ }^{0}$ refer to the back ground electron density of surface sites and bulk(reference) site respectively; $Z_{d}$ and $Z_{i}$ denote the coordination number of the surface site and bulk site atoms respectively; $A_{(x)}$ represents the area per atom near surface; and finally $F_{i}$, the embedding function, generally takes the following form $[20,14]$ :

$$
F(\bar{\rho})=\alpha_{i} E_{c} \bar{\rho} \ln \bar{\rho}
$$

where $\alpha_{i}$ is an artificial parameter. Should a coarse assumption that $\frac{\bar{\rho}_{i}^{x}}{\bar{\rho}_{i}{ }^{0}} \propto \frac{Z_{d}}{Z_{i}}$ be made for unrelaxed mono-atomic lattice with fixed structure, the following expression can be validated by Eq.(2) and Eq.(3):

$$
E_{(x)}^{f}=\sum \frac{E_{c} \frac{Z_{i}-Z_{d}}{Z_{i}}+\alpha_{i} E_{c} \bar{\rho}_{i}{ }^{0} \frac{Z_{d}}{Z_{i}{ }^{2}} \ln \left(\frac{Z_{d}}{Z_{i}}\right)}{A_{(x)}}
$$

60

Note that the $\alpha$ leading section of Eq.(4) is proportional to $1 / Z_{i}^{2}$. This suggests a many-body potential can be reasonably well approximated by 
a two-body equivalent expansion which exploit many coordination shells. Vitos et al [21] proposed that the overlooked many-body terms can be partly "renormalized" into equivalent two-body "effective pair interactions" if many coordination shells are implemented. Da silva et al [10] adopt this concept in their work on the surface energy of 15 different $\mathrm{Cu}$ facets but referred to it as "effective pair potential". Such concept is supported by a linear scaling of surface energy as a function of the number of broken-bonds, which is confirmed in first principle (FP) studies on $\mathrm{Cu}, \mathrm{Pb}[11]$ and $\mathrm{Ni}$ [17] surfaces.

Just as in our latest work, the present model is simplified into a linear combination of effective two-body expansions. Brief calculation suggested the multi-body term in Eq.(4) can be as large as $3 \% E_{c}$ depend on the choice of $\alpha$. Adopting above simplification, Eq.(1) is simply:

$$
\Gamma(\hat{\mathbf{n}})=\frac{1}{2} \sum_{i} f_{\mathbf{b}^{\mathbf{i}}}\left(\mathbf{b}^{\mathbf{i}}\right) \phi_{\mathbf{b}^{\mathbf{i}}}
$$

where $\mathbf{b}^{\mathbf{i}}$ represents a set of crystallographically equivalent vectors. The strength of $\phi_{\mathbf{b}^{\mathbf{i}}}$ terms are estimated via the Rose-Vinet universal potential [22], which takes the following form

$$
\begin{gathered}
E(r)=-E_{c}\left(1+a^{*}+0.05 a^{* 3}\right) e^{-a^{*}} \\
a^{*}=\eta\left(\frac{r}{r_{e}}-1\right) \\
\eta=\sqrt{\frac{9 \Omega B}{E_{c}}}
\end{gathered}
$$

where $E_{c}$ is the atomic cohesive energy, $\Omega$ represents the atomic volume, $B$ denotes the bulk modulus and $r_{e}$ refers to the equilibrium nearest neighbour $\left(1^{\text {st }} \mathrm{NN}\right)$ distance. The width of this potential well is inversely proportional to the dimensionless anharmonicity term $\eta$. The strength of $\phi_{\mathbf{b}^{\mathbf{i}}}$ terms in Eq.(5) is related to the strength ratio between the $i^{\text {th }} \mathrm{NN}$ and $1^{\text {st }} \mathrm{NN}$ interactions, $\frac{\phi_{\mathbf{b}^{\mathbf{i}}}}{\phi_{\mathbf{b} \mathbf{1}}}$, which can be approximated given the interatomic distance ratio between the $i^{\text {th }} \mathrm{NN}$ and $1^{\text {st }} \mathrm{NN}$ interactions, $\frac{\left|\mathbf{b}^{\mathbf{i}}\right|}{\left|\mathbf{b}^{\mathbf{1}}\right|}$, and $\eta$, as following

$$
\frac{\phi_{\mathbf{b}^{\mathbf{i}}}}{\phi_{\mathbf{b}^{\mathbf{1}}}}=\left(1+\eta\left(\frac{\left|\mathbf{b}^{\mathbf{i}}\right|}{\left|\mathbf{b}^{\mathbf{1}}\right|}-1\right)+0.05 \eta^{3}\left(\frac{\left|\mathbf{b}^{\mathbf{i}}\right|}{\left|\mathbf{b}^{\mathbf{1}}\right|}-1\right)^{3}\right) e^{-\eta\left(\frac{\left|\mathbf{b}^{\mathbf{i}}\right|}{\left|\mathbf{b}^{1}\right|}-1\right)}
$$




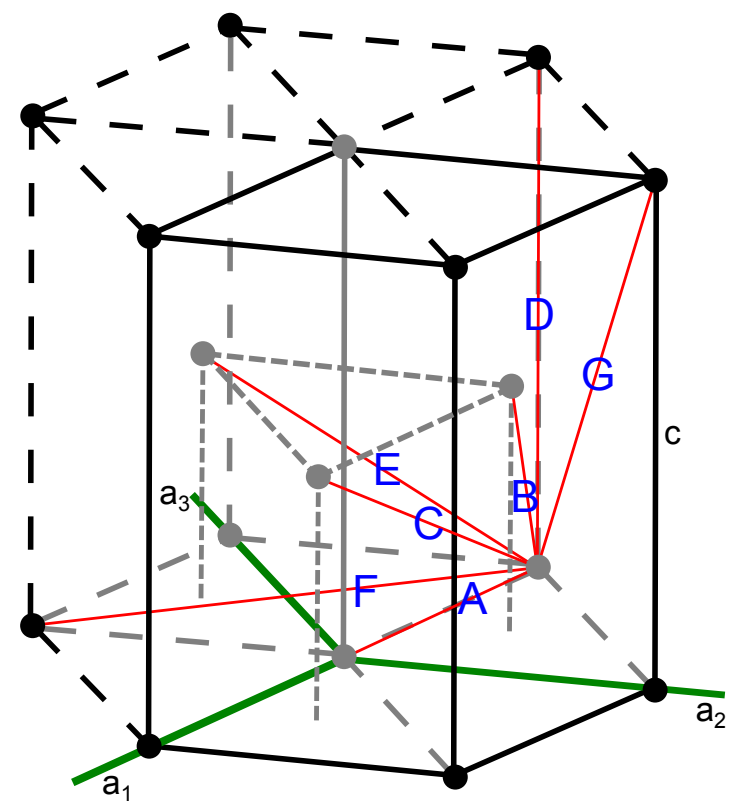

Figure 1: A HCP cell showing the nature of $\mathbf{b}^{\mathbf{i}}$ vectors. The vectors are labelled as referred to in Table 1 and Table 2

For cubic structures, the neighbouring order of a particular set of $\mathbf{b}^{\mathbf{i}}$ is

indifferent from one crystal to another. For HCP structures, on the other hand, the ordering of neighbour is dependent on the actual $c / a$ ratio which varies from one lattice to another, as the hexagonal metals are not exactly "close-packed". In addition, the symmetries presented in HCP lattice are more complicated as compared with cubic systems. In this paper, the 7 nearest $\mathbf{b}^{\mathbf{i}}$ sets are considered in our calculation of surface energy regardless their neighbouring order. The significant $\mathbf{b}^{\mathbf{i}}$ sets are labelled alphabetically, rather than numerically as in our previous work. An example of each $\mathbf{b}^{\mathbf{i}}$ set vector is given in Fig.2. The coordination numbers and bond length (as a function of $a$ and $c$ ) of the $\mathbf{b}^{\mathbf{i}}$ vectors are listed in Table 1 . The 0K zero pressure value of the three input parameters, anharmonicity factor $\eta, 1^{\text {st }} \mathrm{NN}$ bond strength $\phi_{\mathbf{b}^{1}}$ and the relative bond strengths (as compared to $\phi_{\mathbf{b}^{1}}$ ) of $\mathbf{b}^{\mathbf{i}}$ vectors for 13 elemental HCP metals are given in Table 2 .

In a broken-bond method, the excessive energy of a surface with outward normal $\hat{\mathbf{n}}$ is calculated from the strength and number of destroyed bond vectors $\mathbf{b}$ upon the creation of the surface. For a specific $\mathbf{b}$ vector to be 
Table 1: The nature, the corresponding coordination number $Z_{\mathbf{b}^{\mathbf{i}}}$ and the length of significant $\mathbf{b}^{\mathbf{i}}$ vectors presented in HCP lattice

\begin{tabular}{lccc}
\hline & Nature of bond vector & Coordination number $Z_{\mathbf{b}^{\mathbf{i}}}$ & $\left|\mathbf{b}^{\mathbf{i}}\right|$ \\
\hline $\mathbf{b}^{\mathbf{A}}$ & $\{11 \overline{2} 0\}$ & 6 & $a$ \\
$\mathbf{b}^{\mathbf{B}}$ & $\{2 \overline{2} 03\}$ & 6 & $\sqrt{1 / 3 a^{2}+1 / 4 c^{2}}$ \\
$\mathbf{b}^{\mathbf{C}}$ & $\{4 \overline{4} 03\}$ & 6 & $\sqrt{4 / 3 a^{2}+1 / 4 c^{2}}$ \\
$\mathbf{b}^{\mathbf{D}}$ & $\{0001\}$ & 2 & $c$ \\
$\mathbf{b}^{\mathbf{E}}$ & $\{12 \overline{3} 1\}$ & 12 & $\sqrt{7 / 3 a^{2}+1 / 4 c^{2}}$ \\
$\mathbf{b}^{\mathbf{F}}$ & $\{1 \overline{1} 00\}$ & 6 & $\sqrt{3} a$ \\
$\mathbf{b}^{\mathbf{G}}$ & $\{11 \overline{2} 1\}$ & 12 & $\sqrt{a^{2}+c^{2}}$ \\
\hline
\end{tabular}

Table 2: The $0 \mathrm{~K}$ zero pressure input parameters, the calculated dimensionless anharmonicity term $\eta$, the nature $1^{\text {st }} \mathrm{NN}$ bond vector set and the bond strength ratio $\frac{\phi_{\mathbf{b}^{\mathbf{i}}}}{\phi_{\mathbf{b} 1}}$ for 13 elemental HCP metals studied [23]

\begin{tabular}{|c|c|c|c|c|c|c|c|c|c|c|c|c|c|}
\hline Metal & $a(\AA)$ & $c(\AA)$ & $B(\mathrm{GPa})$ & $E_{c}(\mathrm{eV})$ & $\eta$ & $\phi_{\mathbf{b}^{1}}$ & $\frac{\phi_{\mathbf{b}} \mathbf{A}}{\phi_{\mathbf{b}^{1}}}$ & $\frac{\phi_{\mathbf{b}^{\mathrm{B}}}}{\phi_{\mathrm{b}} \mathbf{1}}$ & $\frac{\phi_{\mathrm{b}} \mathrm{C}}{\phi_{\mathrm{b}} 1}$ & $\frac{\phi_{\mathrm{b}} \mathrm{D}}{\phi_{\mathrm{b}} \mathbf{1}}$ & $\frac{\phi_{\mathbf{b}^{\mathrm{E}}}}{\phi_{\mathrm{b}} \mathbf{1}}$ & $\frac{\phi_{\mathbf{b}^{\mathrm{F}}}}{\phi_{\mathrm{b}} \mathbf{1}}$ & $\frac{\phi_{\mathrm{bG}}}{\phi_{\mathrm{b}^{1}}}$ \\
\hline $\mathrm{Be}$ & 2.2856 & 3.5832 & 100.3 & 3.32 & 5.2486 & $\phi_{\mathbf{b}^{\mathrm{B}}}$ & 0.9901 & 1.0000 & 0.3952 & 0.2362 & 0.1485 & 0.1416 & 0.0939 \\
\hline $\mathrm{Mg}$ & 3.2094 & 5.2105 & 35.4 & 1.51 & 7.8289 & $\phi_{\mathbf{b}^{\mathrm{B}}}$ & 0.9995 & 1.0000 & 0.2294 & 0.0861 & 0.0512 & 0.0506 & 0.0206 \\
\hline $\mathrm{Sc}$ & 3.3080 & 5.2653 & 43.5 & 3.90 & 5.5952 & $\phi_{\mathbf{b}^{\mathrm{B}}}$ & 0.9957 & 1.0000 & 0.3741 & 0.2050 & 0.1330 & 0.1288 & 0.0775 \\
\hline $\mathrm{Ti}$ & 2.9506 & 4.6788 & 105.1 & 4.85 & 6.5575 & $\phi_{\mathbf{b}^{\mathrm{B}}}$ & 0.9925 & 1.0000 & 0.2966 & 0.1463 & 0.0851 & 0.0814 & 0.0522 \\
\hline Co & 2.5070 & 4.0690 & 191.4 & 4.39 & 7.3700 & $\phi_{\mathbf{b}^{\mathrm{B}}}$ & 0.9996 & 1.0000 & 0.2555 & 0.1027 & 0.0633 & 0.0626 & 0.0273 \\
\hline $\mathrm{Zn}$ & 2.6649 & 4.9468 & 59.8 & 1.35 & 8.7070 & $\phi_{\mathbf{b}^{\mathbf{A}}}$ & 1.0000 & 0.9837 & 0.1341 & 0.0169 & 0.0252 & 0.0347 & 0.0036 \\
\hline Y & 3.6451 & 5.7305 & 36.6 & 4.37 & 5.5736 & $\phi_{\mathbf{b}^{\mathrm{B}}}$ & 0.9911 & 1.0000 & 0.3693 & 0.2112 & 0.1301 & 0.1240 & 0.0789 \\
\hline $\mathrm{Zr}$ & 3.2312 & 5.1477 & 83.3 & 6.25 & 5.9042 & $\phi_{\mathbf{b}^{\mathrm{B}}}$ & 0.9956 & 1.0000 & 0.3484 & 0.1831 & 0.1160 & 0.1122 & 0.0650 \\
\hline $\mathrm{Tc}$ & 2.7350 & 4.3880 & 297.0 & 6.85 & 8.3263 & $\phi_{\mathbf{b}^{\mathrm{B}}}$ & 0.9955 & 1.0000 & 0.1986 & 0.0734 & 0.0386 & 0.0372 & 0.0153 \\
\hline $\mathrm{Ru}$ & 2.7059 & 4.2815 & 320.8 & 6.74 & 8.5256 & $\phi_{\mathbf{b}^{\mathrm{B}}}$ & 0.9860 & 1.0000 & 0.1832 & 0.0709 & 0.0330 & 0.0309 & 0.0136 \\
\hline $\mathrm{Cd}$ & 2.9788 & 5.6167 & 46.7 & 1.16 & 9.8864 & $\phi_{\mathbf{b}^{\mathbf{A}}}$ & 1.0000 & 0.7399 & 0.0904 & 0.0068 & 0.0128 & 0.0196 & 0.0011 \\
\hline Hf & 3.1946 & 5.0511 & 109.0 & 6.44 & 6.5194 & $\phi_{\mathbf{b}^{\mathrm{B}}}$ & 0.9912 & 1.0000 & 0.2978 & 0.1492 & 0.0859 & 0.0818 & 0.0459 \\
\hline Re & 2.7600 & 4.4580 & 372.0 & 8.03 & 8.7543 & $\phi_{\mathbf{b}^{\mathrm{B}}}$ & 0.9981 & 1.0000 & 0.1818 & 0.0670 & 0.0323 & 0.0315 & 0.0116 \\
\hline
\end{tabular}


broken thus contribute to the calculated surface energy, the following two conditions must be fulfilled: 1) the projection of $\mathbf{b}$ on $\hat{\mathbf{n}}$ must be in the same orientation as $\hat{\mathbf{n}}$, which gives $\hat{\mathbf{n}} \cdot \mathbf{b}>0 ; 2$ ) the parent atom of $\mathbf{b}$ vector must reside within the projection of the bond length on $\hat{\mathbf{n}}$ to be considered as a "surface atom". As such, if atomic volume is denoted as $\Omega$, the areal broken-bond density, $\bar{f}_{\mathbf{b}}$, can be expressed in the following piece-wise manner

$$
\begin{aligned}
& \bar{f}_{\mathbf{b}^{\mathbf{i}}}=\frac{1}{\Omega} \sum_{i} \hat{\mathbf{n}} \cdot \mathbf{b}^{\mathbf{i}} \quad \text { for } \quad \hat{\mathbf{n}} \cdot \mathbf{b}>0 \\
& =0 \quad \text { for } \quad \hat{\mathbf{n}} \cdot \mathbf{b} \leqslant 0
\end{aligned}
$$

Eq.(10) can be directly implemented on cubic but not hexagonal systems. To explain the reason of such difference, the concept of bonding geometry must be introduced. Unlike the atoms in cubic crystals, which have share an indifferent set of significant $\mathbf{b}$ vectors, the atoms in HCP crystals have two different set of significant $\mathbf{b}$ vectors. In other words, an $\mathrm{HCP}$ atom has one of the two bonding geometries associated with its lattice structure. An example on the $\mathbf{b}^{\mathbf{B}}$ vectors is given in Fig.2. It can be seen that the layer A atoms share a set of six $\mathbf{b}^{\mathbf{B}}$ vectors coloured in blue, whereas all layer $\mathrm{B}$ atoms share the other set of $\operatorname{six} \mathbf{b}^{\mathbf{B}}$ vectors coloured in red. This discrepancy occurs when $\mathbf{b}^{\mathbf{B}}$ vectors climb into alternative atomic layers, leading to two distinctive bonding geometries possessed by layer A atoms and layer B atoms respectively. This difference in bonding geometry affect the bond sets $\mathbf{b}^{\mathbf{B}}$, $\mathbf{b}^{\mathbf{C}}$ and $\mathbf{b}^{\mathbf{E}}$. Consider the fact that layer $\mathrm{A}$ and $\mathrm{B}$ each account for $50 \%$ of the total atoms in the lattice, the two bonding geometries will each have $50 \%$ chance to occur for a specific surface atom.

The final expression of areal surface energy takes the following form

$$
\gamma(\hat{\mathbf{n}})=\frac{\phi_{\mathbf{b}^{1} \hat{\mathbf{n}}}}{2 \Omega} \cdot \sum_{i} \mathbf{b}^{\mathbf{i}} \frac{\phi_{\mathbf{b}^{\mathbf{i}}}}{\phi_{\mathbf{b}^{1}}} \quad \text { for } \quad \hat{\mathbf{n}} \cdot \mathbf{b}>0
$$

where $\phi_{\mathbf{b}^{1}}$ represents the $1^{\text {st }} \mathrm{NN}$ bond strength and can be estimated as

$$
\phi_{\mathbf{b}^{1}}=\frac{E_{c}}{\sum_{i} Z_{\mathbf{b}^{\mathbf{i}}} \frac{\phi_{\mathbf{b}^{\mathbf{i}}}}{\phi_{\mathbf{b}^{1}}}}
$$

Eq.(11) is then implemented in a Matlab code to calculate the absolute unrelaxed surface energy and its anisotropy. 


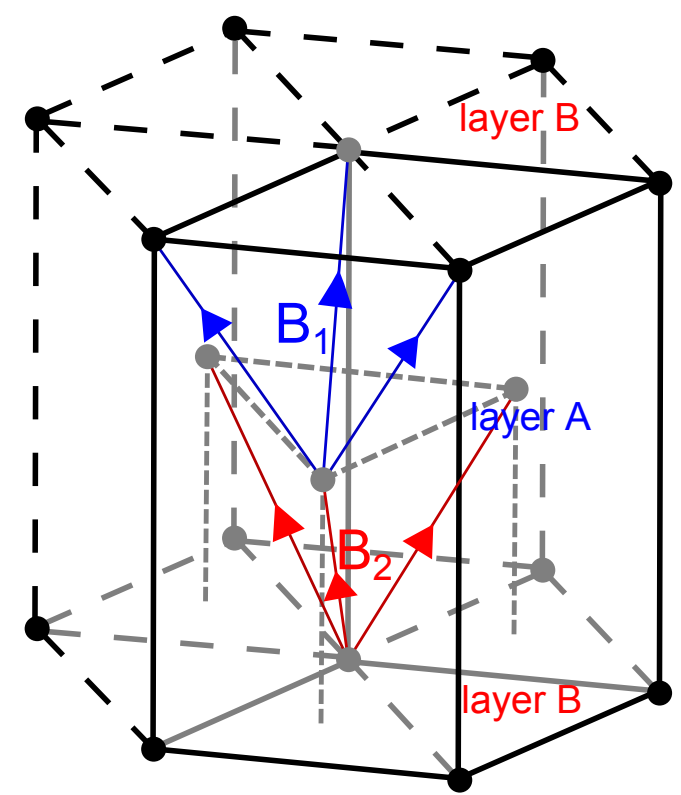

Figure 2: showing the two possible sets of $\mathbf{b}^{\mathbf{B}}$ bond vectors in HCP systems. These are denoted as $\mathbf{b}^{\mathbf{B a}}$ and $\mathbf{b}^{\mathbf{B b}}$ in Table 1. Note the bond vectors are directional

\section{Results and discussion}

Most existing atomistic simulation methods employs simulation cells in which atoms are assigned to specific positions to construct the defect of interest. A disadvantage of such approach is that the simulation cell size will need to increase to accommodate complex surface, thus rendering the whole process more computationally demanding. In a broken-bond method, however, the lattice order is preserved in the inter-atomic vectors, or "bond vectors" as mentioned by Mackenzie [24], whereas the actual coordinates of the atoms are of no importance. On the one hand, the vector based method cannot address issues regarding surface relaxation, meaning it can only estimate the absolute unrelaxed surface energy, but on the other hand, it allows investigation of arbitrarily oriented surfaces, meaning it can assess the full $\gamma$-plot.

Before comparison of our results to experimental findings can be made, the impact of surface relaxation shall be discussed. Although works on this aspect for HCP metals are limited, the quantitative difference between relaxed and unrelaxed surface energy for cubic metals is widely accepted to be orientation-dependent. The degree of this anisotropy is found to vary from 
Table 3: The calculated unrelaxed surface energy of 13 HCP metals. Only (0001) facet and $\gamma_{\max }$ values are listed here. The full anisotropies are given in the complete [0001] stereo projections shown in Fig.3. The results are compared with those from 5 other computational approaches and reported experimental data. It should be known that the experimental values are extrapolated from high temperature multi grain solid-liquid averages. The units are $\mathrm{Jm}^{-2}$.

\begin{tabular}{lllll}
\hline Metals & $\gamma_{(0001)}$ & $\gamma_{\max }$ & Other approaches & Expt. \\
\hline $\mathrm{Be}$ & 2.114 & 2.433 & $1.834^{a}, 1.273^{b}, 1.650^{c}, 1.528^{d}, 2.555^{e}$ & $1.628^{f}, 2.700^{g}$ \\
$\mathrm{Mg}$ & 0.406 & 0.481 & $0.792^{a}, 0.310^{b}, 0.900^{c}, 0.289^{d}, 0.704^{e}$ & $0.785^{f}, 0.760^{g}$ \\
$\mathrm{Sc}$ & 1.150 & 1.322 & $1.834^{a}, 0.706^{b}, 1.355^{c}, 0.816^{d}, 1.114^{e}$ & $1.275^{g}$ \\
$\mathrm{Ti}$ & 1.694 & 1.974 & $2.632^{a}, 1.033^{b}, 1.962^{c}, 1.402^{d}, 1.866^{e}$ & $1.920^{f}, 2.100^{g}$ \\
$\mathrm{Co}$ & 1.986 & 2.332 & $2.775^{a}, 1.162^{b}, 3.056^{c}, 2.857^{d}, 2.297^{e}$ & $2.160^{f}, 2.550^{g}$ \\
$\mathrm{Zn}$ & 0.473 & 0.560 & $0.989^{a}, 0.196^{d}, 1.057^{e}$ & $0.993^{f}, 0.990^{g}$ \\
$\mathrm{Y}$ & 1.066 & 1.232 & $1.506^{a}, 0.623^{b}, 1.001^{c}, 0.737^{d}, 1.008^{e}$ & $1.125^{g}$ \\
$\mathrm{Zr}$ & 1.885 & 2.178 & $2.260^{a}, 0.988^{b}, 2.302^{c}, 1.230^{d}, 1.674^{e}$ & $1.909^{f}, 2.000^{g}$ \\
$\mathrm{Tc}$ & 2.480 & 2.976 & $3.691^{a}, 2.654^{d}, 3.110^{e}$ & $3.150^{g}$ \\
$\mathrm{Ru}$ & 2.479 & 3.002 & $3.928^{a}, 1.281^{b}, 3.191^{c}, 3.362^{e}$ & $3.043^{f}, 3.050^{g}$ \\
$\mathrm{Cd}$ & 0.275 & 0.360 & $0.593^{a}, 0.136^{d}, 0.755^{e}$ & $0.762^{f}, 0.740^{g}$ \\
$\mathrm{Hf}$ & 1.903 & 2.232 & $2.472^{a}, 0.992^{b}, 2.041^{c}, 1.786^{d}, 1.885^{e}$ & $2.193^{f}, 2.150^{g}$ \\
$\mathrm{Re}$ & 2.805 & 3.367 & $4.214^{a}, 1.682^{b}, 3.940^{c}, 2.941^{d}, 3.415^{e}$ & $3.626^{f}, 3.600^{g}$ \\
\hline${ }^{a}$ full charged density (FCD) in the generalized gradient approximation $(\mathrm{GGA})$. Ref.[5] \\
${ }^{b}$ analytic modified embedded-atom method (AMEAM). Ref.[6] \\
${ }^{c}$ MEAM. Ref.[12] \\
${ }^{d}$ empirical electron theory (EET). Ref.[25] \\
${ }^{e}$ equivalent crystal theory (ECT). Ref.[26] \\
${ }_{f}^{f}$ Expt. Ref.[27] \\
${ }_{g}^{g}$ Expt. Ref.[28]
\end{tabular}

one element to another. Also, there seems to be a positive relationship between the magnitude of variation and the roughness of the surface. Recent FP studies on $\mathrm{Cu}$ and $\mathrm{Ni}$ surfaces reported $2.8 \%$ and $7 \%$ maximum variations between relaxed and unrelaxed values respectively [10, 17], whereas both suggested insignificant relaxation on close-packed facets. Consider the experimental values are extrapolated averages from high temperature polygrain solid-liquid observations, we believe a direct comparison between our calculated values and these measurements can still be indicative.

Table 3 shows comparisons of our results on 13 HCP metals with those of 5 other computational methods and 2 sets of experimental findings. It can be seen that our calculated results generally agree with the extrapolated 
measurements, with major discrepancies occur on the three divalent sp metals, namely $\mathrm{Mg}, \mathrm{Zn}$ and $\mathrm{Cd}$, where our results are substantially lower. It is noteworthy that such disagreement is also shared by other geometry based approaches [6, 25], so it would seem broken-bond type models can not describe the behaviours of these metals. The overall strength of anisotropy, $\gamma_{\max } / \gamma_{\min }-1$, is of the $15-21 \%$ range in our results. This range is higher than the $12-16 \%$ range of FCC metals [18], suggesting the surface energy of HCP metals are generally more anisotropic. This comparatively higher anisotropy in HCP metals is also reported from some other computational methods $[25,26,9]$, but the $15-21 \%$ range calculated in this work is much lower than a typical $>30 \%$ range suggested in other theoretical works.

The [0001] stereographic projections of 13 HCP metals studied are tabulated in fig 3, 4 and 5. As a general remark, all projections demonstrated clear six-fold symmetric patterns. The strongest cusp is commonly found at the (0001) pole, followed by a cluster of overlapping minimums located in the vicinity of the $\{10 \overline{1} 1\}$ poles. Weak but distinctive $\{11 \overline{2} 0\}$ cusps are also presented in all figures. The projection patterns are unique for every element, but can be coarsely categorised into three groups. The first group consists of elements Be, Sc, Ti, Y, Zr and Hf, which share regions of favoured energy that stretch inwards along the $\{x 0 \bar{x} y\}$ axis towards the $(0001)$ pole. This group also demonstrates distinctive minimums at the $\{11 \overline{2} 2\}$ and $\{11 \overline{2} 4\}$ poles. Elements $\mathrm{Mg}, \mathrm{Co}, \mathrm{Tc}, \mathrm{Ru}$ and Re form the second group. Unlike the first group, the energetically favoured regions of the second group are somewhat condensed in the middle of the $\{x 0 \bar{x} y\}$ axis. This group shows no detectable minimums on $\{11 \overline{2} 4\}$ but still vague cusps on $\{11 \overline{2} 2\}$ poles. Finally $\mathrm{Zn}$ and $\mathrm{Cd}$, the two elements with highest $c / a$ ratio among all HCP metals studies, are classed as the third group. They present favoured regions that stretch outwards along the $\{x 0 \bar{x} y\}$ axis towards the $\{10 \overline{1} 1\}$ poles, and no longer show distinguishable cusps at $\{11 \overline{2} 2\}$ poles. It is interesting to note that our calculated absolute values of surface energy agree best with experimental measurements for the first group of elements, where the reported experimental averages reside well between $\gamma_{(0001)}$ and $\gamma_{\max }$. The above finding can be explained by the comparatively lower anharmonicity factor $\eta$ possessed by the first group elements. According to Eq.(6) to (9) a lower $\eta$ value suggests more significant further $\mathrm{NN}$ interactions, which lead to reduced error from the $1 / Z_{i}^{2}$ term suggested in Eq.(4). This suggests the elements in the first group may be better described by the current method, and thus the patterns shown in their stereographic projections may be more 
representative for HCP metals.

It is common knowledge that the equilibrium crystal shapes (ECS) of a crystal takes the inner envelope of the corresponding $\gamma$-plot. The stereographic projections given suggest the equilibrium crystal shape of HCP metals generally takes form of a truncated hexagonal bi-prism, as shown in Fig 3 , instead of a full hexagonal prism. The (0001) basal planes are the most energetically favoured thus will always shown, whereas the actual prismatic planes that will occur is differ from one metal to another. The overlapping cusps shown in the middle of the $\{x 0 \bar{x} y\}$ axis suggest faceting on a range of orientations along this axis may have similar likelihood to occur. It should be noted that these findings are fully supported by recent studies on synthesis of HCP nanocrystals [29, 30], in which Ru nanocrystals are produced in both truncated hexagonal bi-prism and hourglass shapes. Apart from the above common feature, a comparatively large inclination angle between the two prismatic facets should be observed for elements in the first group with favoured zones condense towards the (0001) pole, whereas the ECS of Zn and $\mathrm{Cd}$ will be the most prismatic among all. Be, Sc, Ti, Y, Zr and Hf may show $\{11 \overline{2} 2\}$ and $\{11 \overline{2} 4\}$ truncations on the edge of the bi-prism.

\section{Conclusion}

The surface energy anisotropy of HCP metals Be, Mg, Sc, Ti, Co, Zn, Y, $\mathrm{Zr}, \mathrm{Tc}, \mathrm{Ru}, \mathrm{Cd}$, Hf and Re are investigated through a broken-bond type geometric model. Integrating with the Rose-Vinet universal potential, the model enables calculation of the unrelaxed absolute surface energy on arbitrary orientations with only three material constants. The calculated values agrees well with reported experimental extrapolations except for divalent sp metals $\mathrm{Mg}, \mathrm{Zn}, \mathrm{Cd}$, where the calculations are roughly lower by a factor of 2 . The stereographic projections of all 13 metals demonstrate strongest (0001) cusps with an overall anisotropy strength between $15 \%$ to $21 \%$, which is higher than cubic metals. The features shown in projections are unique for every element but generally suggest truncated hexagonal bi-prismatic ECS. Be, Sc, Ti, Y, Zr and Hf have highly weighted further NN contribution by possessing lower $\eta$, thus demonstrate elongated low energy region along the $\{x 0 \bar{x} y\}$ axis towards the (0001) pole. Mg, Co, Tc, Ru and Re show condensed low energy region along the $\{x 0 \bar{x} y\}$ axis between the $\{10 \overline{1} 1\}$ and $\{20 \overline{2} 1\}$ poles. Zn and $\mathrm{Cd}$ also demonstrate low energy zones along the $\{x 0 \bar{x} y\}$ axis but towards the $\{10 \overline{1} 0\}$ poles. Best agreement with experimental findings are found for 
elements with low $\eta$. As such it is believed stereographic projections of low $230 \quad \eta$ elements are more realistic.

\section{Acknowledgement}

The authors are grateful for the financial support from TATA Steel and the Royal Academy of Engineering.

\section{References}

[1] H. Bonzel, Prog Surf Sci 67 (2001) 45-58.

[2] J.-S. Hong, W. Jo, K.-J. Ko, N. M. Hwang, D.-Y. Kim, Philos Mag 89 (2009) 2989-2999.

[3] H. Meltzman, D. Chatain, D. Avizemer, T. M. Besmann, W. D. Kaplan, Acta Materialia 59 (2011) 3473-3483.

[4] M. I. Baskes, R. A. Johnson, Modelling Simul Mater Sci Eng 2 (1994) 147-163.

[5] L. Vitos, A. Ruban, H. Skriver, J. Kollar, Surf Sci 411 (1998) 186-202.

[6] W. Hu, B. Zhang, B. Huang, F. Gao, D. J. Bacon, J Phys-Condensed Matter 13 (2001) 1193-1213.

245 [7] B.-J. Lee, J.-H. Shim, M. Baskes, Phys Rev B 68 (2003) 144112.

[8] J.-M. Zhang, F. Ma, K.-W. Xu, Appl Surf Sci 229 (2004) 34-42.

[9] D.-D. Wang, J.-M. Zhang, K.-W. Xu, Surf Sci 600 (2006) 2990-2996.

[10] J. Da Silva, C. Barreteau, K. Schroeder, S. Blügel, Phys Rev B 73 (2006) 125402.

250 [11] D. Yu, H. Bonzel, M. Scheffler, Phys Rev B 74 (2006) 115408.

[12] J.-M. Zhang, D.-D. Wang, K.-W. Xu, Appl Surf Sci 253 (2006) 20182024.

[13] Y.-N. Wen, J.-M. Zhang, Solid State Commun 144 (2007) 163-167. 
[14] B.-J. Lee, W.-S. Ko, H.-K. Kim, E.-H. Kim, Calphad 34 (2010) 510-522.

[15] X. Wu, R. Wang, S. Wang, Appl Surf Sci 256 (2010) 3409-3412.

[16] M. Fishman, H. L. Zhuang, K. Mathew, W. Dirschka, R. G. Hennig, Phys Rev B 87 (2013) 245402.

[17] W.-B. Zhang, C. Chen, S.-Y. Zhang, J Phys Chem C 117 (2013) 2127421280 .

[18] Y. Luo, R. Qin, Surf Sci 624 (2014) 103-111.

[19] C. Herring, Phys Rev 82 (1951) 87-93.

[20] M. Baskes, Phys Rev B 46 (1992) 2727-2742.

[21] L. Vitos, H. Skriver, J. Kollàr, Surf sci 425 (1999) 212-223.

[22] J. Rose, J. Smith, F. Guinea, J. Ferrante, Phys Rev B 29 (1984) 29632969.

[23] C. Kittel, Introduction to solid state physics, 8th Edition, Wiley, 2004.

[24] J. Mackenzie, A. Moore, J. Nicholas, J Phys Chem Solids 23 (1962) 185-196.

[25] B.-Q. Fu, W. Liu, Z.-L. Li, Appl Surf Sci 255 (2009) 9348-9357.

270 [26] E. Aghemenloh, J. Idiodi, S. Azi, Comp Mater Sci 46 (2009) 524-530.

[27] W. Tyson, W. Miller, Surf Sci 62 (1977) 267-276.

[28] F. R. Boer, Cohesion in metals: transition metal alloys, Cohesion and structure, North-Holland, 1988.

[29] J. Watt, C. Yu, S. L. Y. Chang, S. Cheong, R. D. Tilley, J Am Chem Soc 135 (2013) 606-9.

[30] J. E. Macdonald, M. Bar Sadan, L. Houben, I. Popov, U. Banin, Nat mater 9 (2010) 810-5. 

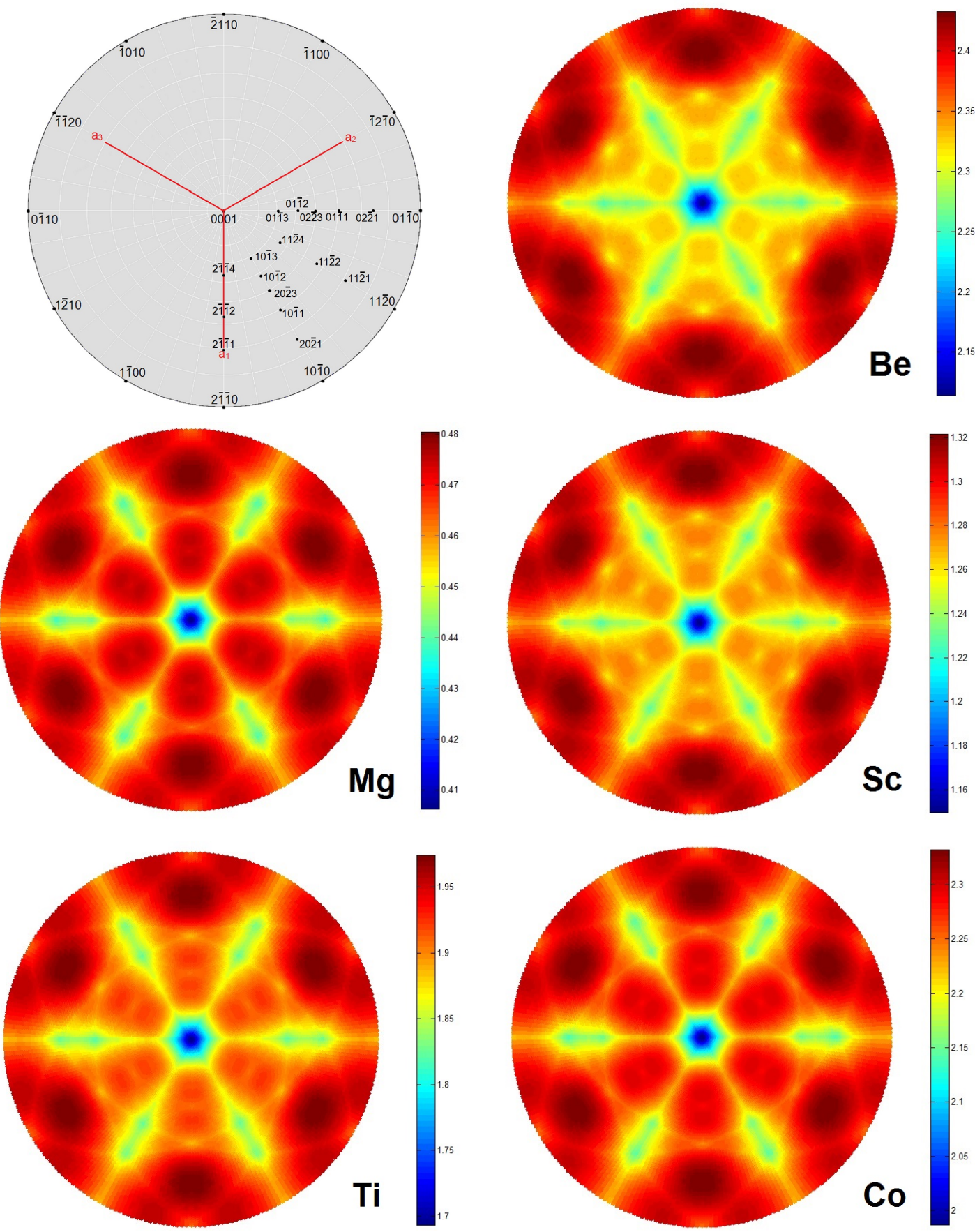

Figure 3: [0001] stereographic projections showing the surface energy anisotropy of Be, $\mathrm{Mg}, \mathrm{Sc}, \mathrm{Ti}$ and Co. Legend is given in upper left. Unit of energy is $\mathrm{Jm}^{-2}$. 

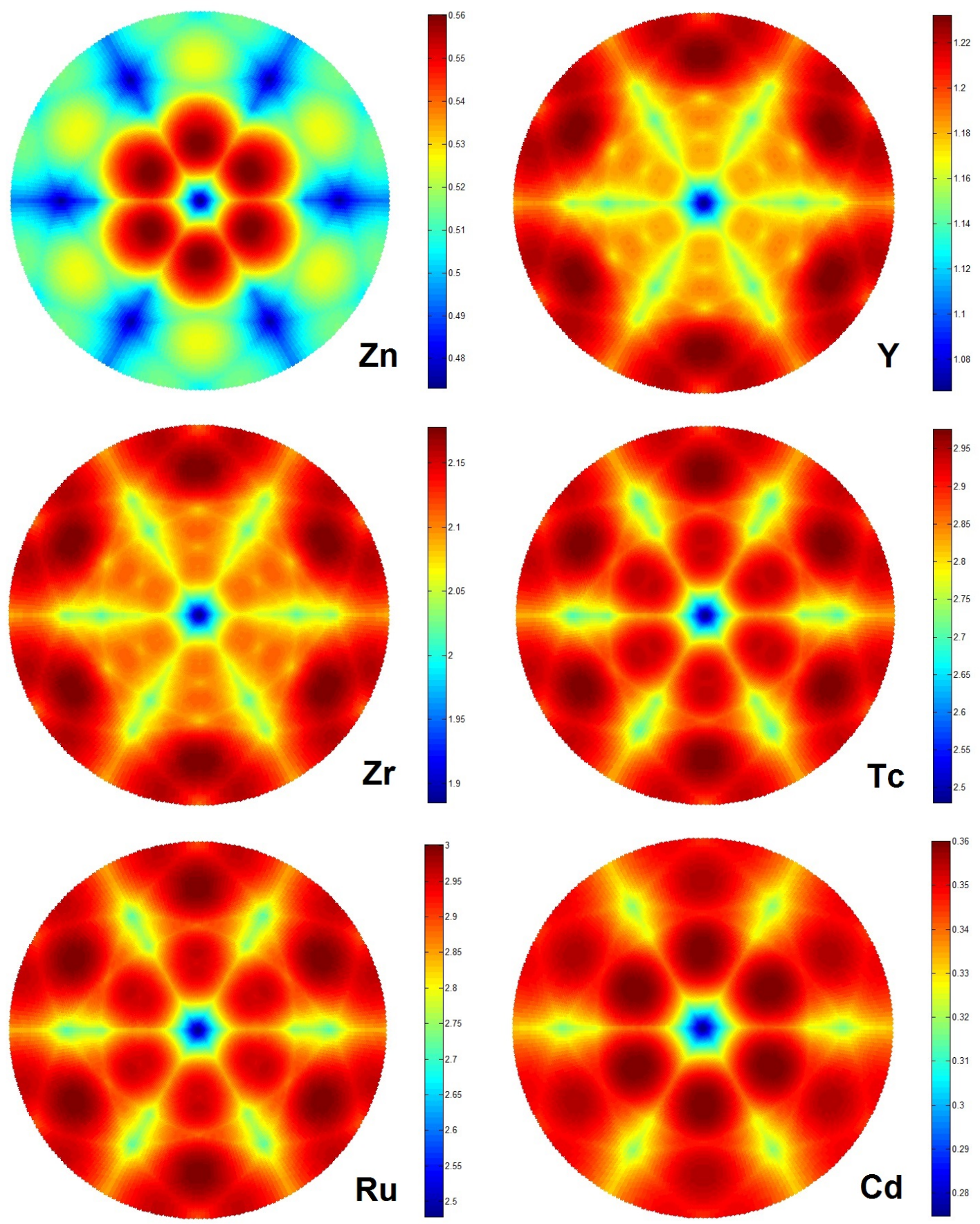

Figure 4: [0001] stereographic projections showing the surface energy anisotropy of Zn, Y, $\mathrm{Zr}, \mathrm{Tc}, \mathrm{Ru}$ and $\mathrm{Cd}$. Unit of energy is $\mathrm{Jm}^{-2}$. 

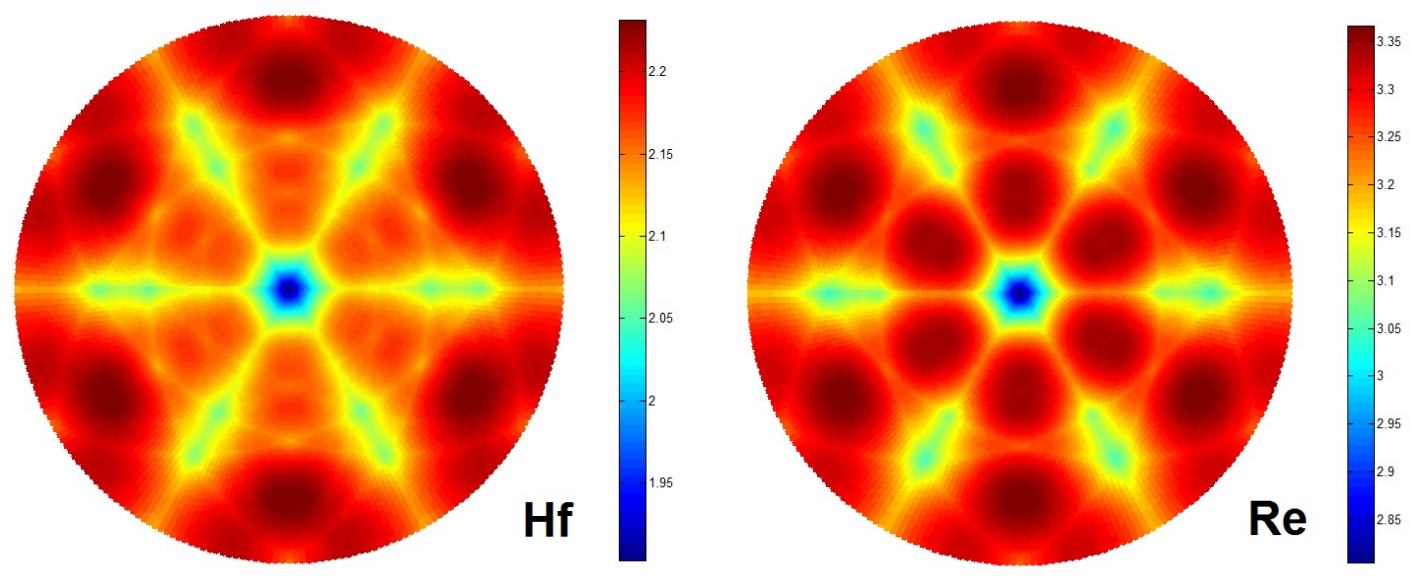

Figure 5: [0001] stereographic projections showing the surface energy anisotropy of Hf and Re. Unit of energy is $\mathrm{Jm}^{-2}$.

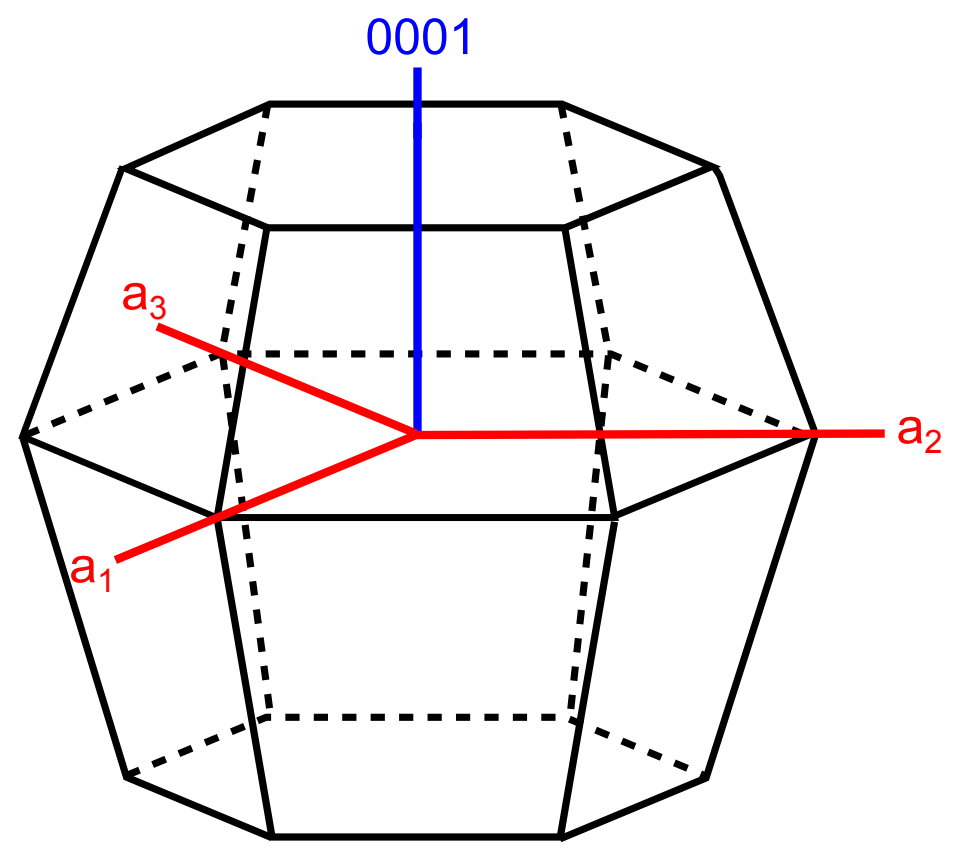

Figure 6: Schematic drawing of the equilibrium crystal shape (ECS) of HCP metals 